\title{
CÓMO AFECTA LA DISCAPACIDAD VISUAL DE UN MIEMBRO DE LA FAMILIA DESDE SU NACIMIENTO. UNA MIRADA DESDE EL TRABAJO SOCIAL
}

\author{
JUDITH INFANTE RUBIO \\ GRADUADA EN TRABAJO SOCIAL \\ UNIVERSIDAD DE LA RIOJA \\ judith.infante@alum.unirioja.es \\ Neus CAPARrós Civera \\ Profesora Titular de Trabajo Social y SERVicios Sociales \\ UNIVERSIDAD DE LA RIOJA \\ caparros@unirioja.es
}

SUMARIO: I. INTRODUCCIÓN. II. TRABAJO SOCIAL Y DISCAPACIDAD. II.1. IMPACTO E IMPORTANCIA DEL NACIMIENTO DE UN NIÑO/A CON DISCAPACIDAD VISUAL. II.1.1. EN LOS PADRES Y MADRES. II.1.2. EN LOS hermanos III. Metodología. III.1. MueStra. III.2. Datos SOCIO DEMOGRÁficos. IV. ReSUltados. IV.1. PADRES Y MADRES. IV.2. HERMANOS V. DisCuSIÓN. VI. CONCLUSIONES. VII. REFERENCIAS BIBLIOGRÁFICAS.

RESUMEN: El nacimiento de un niño/a con discapacidad visual tiene un fuerte impacto en la familia, por lo que va a necesitar apoyo y acompañamiento tanto en el proceso de adaptación a la nueva situación, como en el seguimiento y evolución de la realidad en la que se van a ver inmersos. El texto que aquí se presenta recoge el impacto que tiene en las familias el hecho del nacimiento de ese nuevo miembro con discapacidad visual, a través de un estudio exploratorio sobre 19 familias, seleccionadas por un muestreo por conveniencia que, de una u otra manera, han establecido contacto con una de las instituciones, la ONCE, que se dedica a la intervención con personas con discapacidad visual. Se verá si el enfoque dado a las intervenciones es concéntrico o no, de ahí que se aborde la situación de las personas con discapacidad visual, no como un hecho aislado, sino como algo inmerso en una realidad más amplia: la familia, en lo que a progenitores y hermanos/as se refiere.

PALABRAS CLAVE: Discapacidad visual, Trabajo Social, familia, intervención, acompañamiento. 


\title{
HOW VISUAL IMPAIRMENT AFFECTS A FAMILY MEMBER FROM BIRTH. A VIEW FROM SOCIAL WORK
}

\begin{abstract}
The birth of a child with visual impairment has a strong impact on the family itself, so it/ they will need support and accompaniment both in the process of adaptation to the new situation, and in the monitoring and evolution of the reality they are going to be immersed. "The aim is to show what interventions are carried out by the SC, and how they work with parents, brothers, and sisters of children with visual impairment." The text presents the impact that the birth of this new member with visual impairment represents on families, through an exploratory study of 19 families selected by a convenience sampling that, in one way or another, they have established contact with one of the institutions, such as the ONCE, which is dedicated to intervening with families who have one of their members with visual impairment. It will be seen if the approach given to interventions in families with a member with visual impairment is concentric or not, hence the situation of people with visual impairment is approached, not as an isolated event, but as something immersed in a reality broader: the family, as far as parents and siblings are concerned.
\end{abstract}

KEY WORDS: Visual disability, Social Work, family, intervention, accompaniment.

\section{Introducción}

La profesión del Trabajo Social a lo largo de su historia se ha ocupado de familias en situación de vulnerabilidad, de emergencia social o de exclusión y del colectivo de las personas con discapacidad como una de sus prioridades y preocupaciones. Fue la propia Mary E. Richmond quien ya consideraba a la familia como la primera comunidad en la que una persona se desarrollaba y quien puso de manifiesto la gran importancia de las relaciones entre sus miembros para su correcto desarrollo y evolución. De ahí que, desde el Trabajo Social también se atienda a las familias de las personas con discapacidad visual pues, como se detallará más adelante, pueden llegar a sufrir un gran impacto ante el nacimiento de un hijo/a con este tipo de discapacidad.

Esta concepción es compartida por la Fundación Derecho y Discapacidad, la cual sostiene que la discapacidad no puede ser tomada como una circunstancia personal e individual, sino que debe entenderse como un fenómeno social en el que participan también la sociedad y, especialmente, la familia (Fundación Derecho y Discapacidad, 2015).

España es un país tradicionalmente familiar, donde, por excelencia, la familia es la institución encargada de proporcionar los apoyos necesarios para el desarrollo de sus miembros y, además, constituye el primer núcleo de socialización de la persona (Palacios, 2008), por lo que el texto que aquí se presenta recoge el impacto que representa en las familias el hecho del nacimiento de un nuevo miembro con discapacidad visual. El análisis se realiza sobre las familias que de una u otra manera han establecido contacto con una de las instituciones, como es el caso de la ONCE, que se dedica a la intervención con familias que tienen a alguno de sus miembros con discapacidad visual.

Veremos si el enfoque dado a las intervenciones en familias con algún miembro con discapacidad visual es concéntrico o no, de ahí que se aborde la situación de las personas con discapacidad visual, no como un hecho aislado, sino como algo inmerso en una realidad más amplia: la familia, en lo que a progenitores y hermanos/as se refiere.

Estudiar y atender las familias que tienen uno o varios hijos/as con discapacidad visual radica principalmente en dos aspectos. Primero, en observar la importancia del conocimiento de la realidad 
social que enmarca y caracteriza al Trabajo Social, y segundo, en la necesidad de poder reconsiderar y reencauzar las intervenciones, hacia este colectivo y desde esta profesión hacia un modelo más global.

La finalidad de este estudio es analizar las intervenciones llevadas a cabo desde el Trabajo Social con las familias con hijos/as con discapacidad visual. Para ello, se han fijado los siguientes objetivos.

1. Estudiar el impacto psicosocial producido en el seno de las familias ante la aparición de una discapacidad visual por parte de alguno de los descendientes.

1.1. Conocer el perfil y las características de las familias con uno o varios hijos/as con discapacidad visual.

1.2. Examinar las diferencias en los distintos miembros de la familia con respecto al alcance del impacto de la discapacidad visual.

1.3. Plasmar y dar visibilidad a las situaciones y necesidades reales de las familias con uno o varios hijos/as con discapacidad visual.

2. Profundizar sobre el papel del trabajador/a social como profesional en la intervención con las familias ante la aparición de una discapacidad visual por parte de alguno de los descendientes.

2.1. Poner de manifiesto las áreas prioritarias de intervención desde el Trabajo Social para resaltar aquellos aspectos que deberían guiar la praxis de dicha profesión.

\section{Trabajo social y discapacidad}

La discapacidad es un fenómeno que, especialmente en este último siglo, está adquiriendo gran importancia en la sociedad, en las políticas sociales, en las prestaciones y en la protección social. (Muyor, 2011). Esto ha traído consigo una nueva visión sobre las personas con discapacidad, lo que ha permitido plantar cara a las viejas concepciones y luchar por la integración de estas personas en la sociedad (Egea y Sarabia, 2004).

Según Del Fresno y Segado (2013), la familia puede considerarse como la primera comunidad de una persona, el contexto en el que se desarrolla y donde se satisfacen las primeras necesidades de las personas. Por ello, desde sus orígenes, las familias multiproblemáticas o en conflicto han sido un objeto primordial de investigación e intervención desde esta profesión (Lorenzo, 2015).

En este sentido, escuchar y comprender a los familiares de las personas con discapacidad resulta imprescindible en los procesos de intervención social (Del Fresno y Segado, 2013), pues subscribe una "perspectiva clarificadora y una mirada innovadora a la resolución de los problemas sociales, más allá de la lógica de los recursos” (Lorenzo, 2015, pág. 15).

Pese a la importancia de la familia, en ocasiones, la línea de actuación profesional puede verse acaparada por un enfoque concéntrico, centrado solo en la persona con discapacidad, sus necesidades, sentimientos y problemas. Así, la persona puede no ser vista como una parte de un sistema, un contexto o un entorno, sino como un ser individual, desvinculado de la familia o de la comunidad, lo que puede desembocar en la desatención o ignoración de las necesidades familiares (Egea y Sarabia, 2004). 
Como consecuencia de este enfoque concéntrico han tenido lugar grandes progresos en la atención de las personas con discapacidad, pero también se ha producido una "miopía profesional", en palabras de Kew (1978, pág. 13), una perspectiva distorsionada que ha ignorado una parte de la discapacidad visual en sí, como es la familia de la persona.

De esta manera, este enfoque individualista debería dejarse atrás para dar paso a una mirada más global en la que la familia sea vista como un grupo en el que tiene lugar un acontecimiento especial (el nacimiento del niño/a con discapacidad) que ocasiona un conjunto de nuevas necesidades y que puede llegar a provocar un desequilibrio de las fuerzas familiares. Desde esta perspectiva, el niño/a con discapacidad visual se aleja del foco de acción, el cual pasa a la familia como grupo social.

En el III Congreso Nacional de Familias, organizado por la Confederación Española de Organizaciones en favor de las Personas con Discapacidad Intelectual (FEAPS, 2006) algunos miembros de familias con personas con discapacidad sentenciaron que "su calidad de vida había mejorado, pero de forma indirecta: a través de los apoyos que sus hijos habían recibido y no tanto por los que habían recibido como familia" (FEAPS, 2006, pág. 43) y que es el momento de "dar un salto cualitativo, incluyendo realmente a las familias como receptoras de atención y apoyo específico" (FEAPS, 2006, pág. 44).

Hoy en día, la familia sigue desempeñando en España un papel protector, un rol de atención, apoyo y cuidado a las personas que, de un modo u otro, pueden llegar a tener algún tipo de dependencia, como es el caso de las personas con discapacidad.

Pese a que este "rol protector" siempre ha estado presente, las políticas sociales y las estrategias integrales de apoyo a la familia en España tienen un corto desarrollo, pues no es hasta finales del S.XX, con la Ley de Bases de la Seguridad Social (1963), cuando comienzan a tomar relevancia aquellas familias con miembros con discapacidad (IMSERSO; Ministerio de Trabajo y Asuntos Sociales; y Secretaría de Estado de Servicios Sociales, Familias y Discapacidad, 2004).

En este momento, los valores sociales giran en torno a que las familias deben recibir los apoyos necesarios para conservar a la persona con discapacidad en el hogar, extendiendo la atención social a todas las personas que conforman la familia (Heward, 1998).

Desde el Trabajo Social, los dos pilares de atención con las familias con miembros con discapacidad son la capacitación y la ayuda, y siempre resaltando la importancia de la promoción, el desarrollo y el bienestar de las personas con discapacidad (Silva, 2012).

\section{II.1. Impacto e importancia del nacimiento de un niño/a con discapacidad visual}

\section{II.1.1. En los padres y madres}

El nacimiento de un hijo/a es un hito importante en la vida de las personas, por lo que las expectativas sobre ello son, generalmente, muy elevadas. Cuando una pareja espera a un bebé, esta suele volcar todas sus esperanzas en la criatura y se forma una idea del niño/a esperado/a. Así, antes de nacer, el bebé es "todo menos un bebé", pues se ha convertido en un "tópico en el que se depositan los deseos de los padres” (P. Ben Soussan, 1999; citado en Díaz, 2004, pág. 133).

Desde este punto de vista, el nacimiento del niño/a supone la ruptura con ese bebé imaginario, ruptura que podría llegar a ser más adversa si se trata de un bebé con discapacidad visual (Kew, 1978). Así, es comprensible que el nacimiento de un hijo/a con discapacidad visual sea un potencial factor desequilibrante del grupo familiar y un posible desencadenante de una crisis familiar. 
El nacimiento de un niño/a con discapacidad visual puede valorarse por los progenitores como una situación estresante, ya sea de daño, amenaza o pérdida, que puede desencadenar en una crisis emocional familiar al valorarse que no se tienen los recursos y habilidades necesarios para afrontarla. No obstante, el contexto familiar también es un factor influyente en este proceso, de manera que algunos aspectos como las relaciones de pareja, la dinámica familiar, el nivel sociocultural, la existencia de apoyos o el orden de nacimiento del bebé pueden cobrar especial relevancia.

Siguiendo a Díaz (2004), Freixa (1993) y Lucerga y Sanz (2004), entre otros, pueden distinguirse varias etapas o estadios en el proceso de aceptación del hijo/a con discapacidad visual. En un primer momento, los padres -especialmente la madre- pueden atravesar un periodo de crisis emocional acompañado de sentimientos como la conmoción, la negación, la sorpresa o la ira. Sentimientos que, tras un periodo de desorganización y readaptación emocional, pueden irse transformando en otros como la vergüenza, la culpa, el rechazo o la sobreprotección. Finalmente, tras un periodo de duelo por la pérdida del niño/a esperado/a y la asunción de un hijo/a real con una discapacidad visual, se llega a una tercera etapa en la que los progenitores aceptan a su bebé.

Algunos de los sentimientos y emociones que suelen aparecer en los padres/madres a lo largo del proceso de aceptación son la conmoción, la agresividad, el rechazo, la pena, la culpa, sentimientos, que representan tan solo una parte del conjunto de emociones que pueden pasearse por la mente de los ascendientes, sentimientos que de alguna manera, marcan las fases por las que los progenitores -los cuales no tienen por qué hacerlo al mismo ritmo- van avanzando en el proceso de aceptación, adaptación y normalización de su nueva situación.

Según algunos autores/as, el proceso de adaptación al nacimiento de un hijo/a con discapacidad puede estar íntimamente ligado e influido por las motivaciones -generalmente inconscientes- que están detrás del deseo de tener un bebé. Brazelton y Cramer (1993), citados en Lucerga y Sanz (2004).

Las necesidades de un hijo/a con discapacidad visual son diferentes a las que pueda presentar un niño/a que no la tenga. Esta necesidad de atención especial puede provocar que los padres/madres deban pasar, al menos al principio, más tiempo en casa con su hijo/a, pudiendo desembocar en un aislamiento, un deterioro de las relaciones sociales y un profundo sentimiento de soledad (Olshansky, 1962). Además, este aislamiento, según Kew (1978), puede ser inducido por los sentimientos de vergüenza que pueden sentir algunos padres/madres cuando, al salir de casa, deben "exhibir" a sus hijos/as. A este respecto, Sheila Hewett (1970) añade que este aislamiento suele manifestarse más en las zonas rurales debido, en primer lugar, a la dificultad para entablar amistades con personas que se hallen en una situación similar, y, en segundo lugar, a las limitaciones del transporte.

\section{II.1.2. En los hermanos}

El nacimiento de un hijo/a es un hito que marca un antes y un después en la vida de sus progenitores, algo que es innegable también en el caso de sus hermanos/as, pues la llegada de un hermano/a supone un cambio muy significativo en su vida.

La relación fraternal puede llegar a constituir una parte básica dentro del desarrollo de la socialización de un niño/a, pues es una de las primeras relaciones interpersonales que se mantienen. A través de esta interacción, la cual se establece de forma continua y a largo plazo (Mendoza, 2005), los niños/as se desarrollan recíprocamente, "aprenden destrezas sociales, aprenden a compartir, a rivalizar, a demostrar lealtad, a expresar sentimientos”, etc. (Mendoza, 2005, pág. 32). De manera que puede constituir un papel fundamental en el desarrollo del área socioemocional de ambos/as. 
No obstante, el posible tambaleo del equilibrio familiar ante el nacimiento de un niño/a con discapacidad visual también puede afectar a la relación fraternal ya que el tiempo y la energía que los padres/madres dirigen al hijo/a con discapacidad visual puede ir de la mano de una disminución del tiempo y energía empleado hacia el otro hijo/a (Segura y Torras, 2014), de manera que este último/a puede sentir que ha "pasado a un segundo plano". Para contrarrestar esta situación, el niño/a sin discapacidad suele reaccionar de dos modos: creando una rivalidad hacia su hermano/a o tratando de llamar la atención de sus progenitores (Freixa, 1993; Mendoza, 2005; Ponce, 2008; Segura y Torras, 2014; etc.).

En estas situaciones y para evitar este tipo de conductas, los padres/madres suelen tratar de establecer un equilibrio en el que el tiempo pasado con un hijo/a se compense con el tiempo pasado con el otro/a (Lucerga y Sanz, 2004).

Se considera importante destacar aquí que no todos los sentimientos que puede llegar a producir la llegada de un hermano/a con discapacidad visual deben ser negativos, ya que también puede tener efectos muy positivos "para la vida cotidiana y el futuro de los niños y su familia como son la madurez, la responsabilidad, el altruismo, la tolerancia, la confianza en sí mismos y la independencia." (Díaz, 2004, pág. 141).

\section{Metodología}

Debido a la complejidad que supone estudiar un hecho tan trascendente como el nacimiento de un hijo/a con discapacidad visual, y a su doble vertiente objetivo-subjetiva, se ha decidido poner en práctica el pluralismo metodológico y recabar datos tanto cuantitativos como cualitativos, unificando así la dimensión o perspectiva humanista y la cientifista.

De esta manera, se considera que la triangulación es el método más oportuno para la realización de este estudio.

El presente trabajo se presenta, en primer lugar, como un estudio exploratorio sobre la realidad de las familias ante el nacimiento de un hijo/a con discapacidad visual y su impacto; verificar la viabilidad de una investigación de este tipo y ensayar la metodología puesta en práctica. En segundo lugar, también es un estudio descriptivo, pues trata de plasmar algunos aspectos como el impacto vivenciado por las familias, sus características sociodemográficas o las áreas familiares en las que ha podido llegar a incidir este acontecimiento. Y, por último, se configura como un estudio evaluativo de las intervenciones llevadas a cabo desde el Trabajo Social en este ámbito, para comprobar su futura viabilidad en otros estudios.

De acuerdo con el objetivo de revelar las diferentes percepciones entre los distintos miembros de la familia con respecto al alcance del impacto de la discapacidad visual, se han realizado dos encuestas; una para los hermanos/as y otra para los progenitores.

Ambas encuestas están dividas en bloques temáticos, recogiendo el primero de ellos datos demográficos de la unidad familiar y el resto el impacto que ha tenido el nacimiento de un miembro con discapacidad visual. En ambas encuestas se han utilizado las escalas de Guttman y la de tipo Likert. 


\section{III.1. Muestra}

Para llevar a cabo el estudio, se contactó con la ONCE en La Rioja, para acceder a las familias que tuvieran un miembro con discapacidad visual. De esta manera se proporcionaba un marco (base de sondeo) actualizado y completo en el que figuran los afiliados/as con una discapacidad visual congénita (todos ellas y sin duplicaciones), junto con los datos de localización necesarios, por lo que constituía un marco válido y fiable. La existencia de este marco configura la naturaleza de la muestra como probabilística.

En el presente estudio se acotaron 19 personas (19 familias) con un universo poblacional de 52 personas, de los cuales 37 eran progenitores y 15 hermanos y hermanas.

Esto responde a que el objeto de estudio son aquellas familias en las que se produce el nacimiento de un niño/a con discapacidad visual, condición en la que reside el primer criterio de acotamiento. Al ser una población finita -ya que es inferior a 10.000 unidades- y un universo reducido -ya que es inferior a 500 unidades-, podría haberse estudiado la totalidad de las familias, no obstante, siguiendo un procedimiento de muestro razonado y opinático, se redujo las familias objeto de estudio atendiendo a la edad de los afiliados.

Para estudiar la representatividad estadística de la muestra se utilizó la siguiente fórmula:

$$
E M=z_{\alpha / 2} \cdot \sqrt{\frac{\widehat{p}(1-\widehat{p})}{n}}
$$

Donde EM es el error muestral que se está dispuesto a admitir; $\alpha$ el nivel de significación; $\mathbf{z}_{\alpha / 2}$ el valor que deja a la derecha una probabilidad de $\alpha / 2$; $\hat{\mathrm{p}}$ la proporción poblacional; y n el tamaño de la muestra.

Así, para un nivel de confianza $(1-\alpha)$ del $99 \%$, y habiendo estudiado a las familias de 19 afiliados $(\hat{\mathrm{p}}=0.61)$, se obtiene un error muestral de

$$
\begin{aligned}
& \mathrm{EM}=\mathrm{z}_{\alpha / 2} \cdot \sqrt{\frac{\widehat{\mathrm{p}}(1-\widehat{\mathrm{p}})}{\mathrm{n}}}=\mathrm{z}_{0.01 / 2} \cdot \sqrt{\frac{\frac{19}{31}\left(1-\frac{19}{31}\right)}{31}}=2.575 \cdot \sqrt{\frac{0.23725}{31}}=2.575 \cdot 0.08748 \\
& \mathrm{EM}=0.22526
\end{aligned}
$$

De esta manera, se consideró que la muestra escogida era estadísticamente representativa.

\section{III.2. Datos socio demográficos}

De la muestra 18 fueron hombres y 19 mujeres, puesto que una de las familias estaba formada tan solo por un hijo y su madre. Esta familia coincide con el caso de viudedad. A este respecto es destacable la reconstrucción de las familias, pues en el $21.05 \%$ de ellas, el progenitor masculino es el padrastro, no el padre. No obstante, se han considerado unidades de análisis válidas puesto que todos ellos han estado presentes en el nacimiento y desarrollo del niño/a con discapacidad. De los 4 casos en los que se ha producido una reconstrucción familiar, 3 fueron a causa del nacimiento del niño/a con discapacidad.

De las 19 familias, 6 estaban formadas por dos progenitores y un hijo/a; 1 por una madre y un hijo; 9 por dos progenitores y dos hijos/as; y 3 dos progenitores y tres hijos/as. Así, el número 
medio de hijos/as por familia es de 1.79, con una cuasidesviación típica de 0.71 y el número medio de personas que forman el núcleo familiar es de 3.74 con una cuasidesviación típica de 0.81 .

Atendiendo al número de personas con discapacidad en las familias, se observa que ningún progenitor presenta discapacidad, pero sí existe una familia que tiene dos hijos/as con discapacidad (uno visual y otro auditiva).

Por otro lado, a través del estudio de la localización de la residencia de las familias, se observa que un $57.89 \%$ de ellas reside en pueblos de La Rioja. mientras que las restantes 8 familias residen en la ciudad de Logroño.

De los 19 niños/as con discapacidad visual, un 42.11\% son varones y un $57.89 \%$ son mujeres. Sus edades están comprendidas entre los 3 y los 10 años, siendo la media de edad de 7.52 con una cuasidesviación típica de 1.98. En lo que respecta a la educación, un 47.37\% de los niños/as se encuentra en centros normalizados, mientras que $10 \mathrm{de}$ ellos/as cursan sus estudios en centros de educación especial. Todos/as ellos/ellas son beneficiarios/as de ayudas de la ONCE.

El hecho de que más de un $50 \%$ de los niños/as objeto de estudio curse sus estudios en centros de educación especial se debe a que tienen otras discapacidades. De ese $52.63 \%$ de los niños/as, un $70 \%$ presentan una discapacidad física, un $10 \%$ intelectual y un $20 \%$ diversas discapacidades.

La aparición y desarrollo de la discapacidad visual del $21.05 \%$ de los niños/as fue repentina, mientras que la del $78.95 \%$ formó parte de un proceso progresivo.

En cuanto al orden de nacimiento, se ha observado que 13 de los 19 fueron concebidos en primer lugar; 4 en segundo lugar; y 1 en tercer lugar. Se han contabilizado 7 casos en los que el nacimiento de un niño/a con discapacidad visual ha conllevado el planteamiento, retraso u olvido de un siguiente embarazo.

En cuanto a las características de los progenitores, se han estudiado un total de 19 madres $(51.35 \%$ ) y 18 padres (48.65\%). La edad media actual de las madres ha oscilado entre los 23 y los 52 años $(x=37.12 ; \mathrm{S}=8.85)$ y la de los padres entre los 25 y los 56 años $(\mathrm{x}=39.59 ; \mathrm{S}=10.32)$. Así, mientras la edad media actual se posiciona en 37.12 años, la edad media en el momento del nacimiento del con discapacidad visual se situaba en los 29.58 .

Atendiendo al estado civil, un $40.54 \%$ están casados/as, un $32.43 \%$ unidos/as de manera consensuada, un $2.7 \%$ en estado de viudedad, y un $24.32 \%$ con pareja.

Atendiendo al lugar de residencia, un $61.54 \%$ de las personas estudiadas, residen en pueblos de La Rioja, mientras que un $38.46 \%$ tienen su residencia habitual en la capital de la Comunidad Autónoma.

En cuanto a las creencias, el $54.05 \%$ de los progenitores se considera no creyente; el $21.62 \%$ católico; y el $24.32 \%$ creyente de otra religión.

Atendiendo a la formación académica y a la ocupación, todos los progenitores tienen estudios de diferentes niveles. 6 de ellos estudios secundarios, 14 estudios universitarios, 8 estudios de grado medio y 9 estudios de grado superior. Pese a ello, un $29.73 \%$ se encuentra desempleado; un $13.51 \%$ se constituye como trabajador/a del hogar y un $2.7 \%$ como pensionista. De aquellos con empleo remunerado, un $5.41 \%$ son trabajadores por cuenta propia y un $48.65 \%$ por cuenta ajena.

Por otra parte, destaca que ninguno de los progenitores tiene una discapacidad de ningún tipo.

En cuanto a las características sociodemográficas de los hermanos/as, se encuentra una predominancia masculina: de los quince participantes, solo cinco eran mujeres (33.34\%). 
Los datos referentes al estado civil muestran que un $6.67 \%$, forma parte de una unión consensuada; un $6.67 \%$ tiene pareja y un $86.67 \%$ no tiene pareja. Esto puede deberse a la corta edad de los participantes, pues ha oscilado entre los 4 y los 26 años, siendo la edad media de 12.33 años, con una cuasidesviación típica de 6.83. Este factor también influye en los resultados obtenidos con respecto a la ocupación, pues el $86.67 \%$ son estudiantes y el $13.33 \%$ trabajadores/as por cuenta ajena.

En cuanto a la educación, el 20\% de los hermanos/as se encuentra en educación infantil; el $40 \%$ en primaria; el $20 \%$ en secundaria; el $6.67 \%$ tiene estudios universitarios y un $13.33 \%$ de grado superior.

También se ha analizado la edad de los hermanos/as en el momento del nacimiento del hermano/ a con discapacidad, obteniendo que la edad media en aquel momento era de 6.9 años con una cuasidesviación típica de 4.79. La diferencia media de edad con el hermano/a con discapacidad es de 5.60, oscilando entre los 0 y los 17 años y con una cuasidesviación típica de 4.47 .

Por otro lado, atendiendo al orden de nacimiento, un $66.67 \%$ de los hermanos/as son mayores que el niño/a con discapacidad visual; un $26.67 \%$ son menores; y un $6.67 \%$ posee la misma edad, puesto que son gemelos. Además, tras el estudio del número de hermanos/as se observa que el $60 \%$ de los casos tienen un hermano/a y el $40 \%$ de los casos tienen dos.

En cuanto a las creencias, 4 de los 15 hermanos/as se consideran católicos/as, 3 no creyentes y 8 no han sabido o querido contestar a la pregunta.

\section{Resultados}

\section{IV.1. Padres y madres}

Uno de los objetivos de este trabajo era estudiar el papel del trabajador/a social como profesional de la intervención en estas familias y reflexionar sobre las intervenciones que lleva a cabo. Por ello ha sido una de las cuestiones dirigidas a los progenitores.

De las 19 familias estudiadas, el 100\% ha afirmado haber tenido contacto, a nivel profesional, con algún trabajador/a social. De ellas, ante la pregunta de si "este contacto ha sido a causa de la discapacidad visual de su hijo/a", un $94.74 \%$ (18 familias) han respondido que sí, siempre; un 5.26\% que generalmente sí y un $0 \%$ ha respondido que no. Ese mismo $94.74 \%$ ha afirmado que no han sido, a su parecer, objetos propios de la intervención de los trabajadores/as sociales que les han atendido.

Según 16 familias (84.21\%), el trabajador/a social que les ha atendido siempre ha estado centrado en el hijo/ a con discapacidad, según 2 de ellas, esto ha ocurrido con mucha frecuencia y según 1 , con bastante frecuencia.

En el momento del descubrimiento de la discapacidad visual de su hijo/a, un 63.16\% no sabe si recibió información, orientación o asesoramiento por parte de un trabajador/a social, un $31.58 \%$ afirman que fue así, y un 5.6\% lo niegan. No obstante, ante la pregunta de si recibieron algún "apoyo por parte de un trabajador/a social" tras conocer la discapacidad visual del hijo/a, un $84.21 \%$ afirma que sí.

En cuanto a las entidades o asociaciones que han prestado apoyos a las familias, destacan ASPACE (5.26\%); ONCE (100\%); La Rioja sin Barreras (31.58\%); y la Asociación de personas sordas de La Rioja $(5.26 \%)$. 
En cuanto a la calidad de los apoyos recibidos, destaca la insatisfacción generalizada de las familias, pues un $52.63 \%$ los califica como incompletos y escasos y un $15.79 \%$ como mejorables. Estas respuestas se justifican en que, según los progenitores, los apoyos recibidos son principalmente de tipo económico y, según un $89.47 \%$ de las familias, necesitaban mayores apoyos de tipo social.

Además, un $47.37 \%$ de las familias sostiene que las políticas sociales actuales no se ajustan a las necesidades de las demandas de las familias con hijos/as con discapacidad, y un $42.11 \%$ sostiene que a veces sí se ajustan. Por ello, ante la pregunta de si consideran que las intervenciones profesionales desde el Trabajo Social deben centrarse más en los familiares de las personas con discapacidad visual, un $89.48 \%$ ha afirmado que sí.

Otra de las cuestiones abordadas en la encuesta realizada a los progenitores han sido los sentimientos surgidos ante el nacimiento de un hijo/a con discapacidad visual. Estos datos se han recogido a través de una escala de Guttman.

Tras el nacimiento del bebé y el diagnóstico de la discapacidad visual, todos los progenitores pasaron un tiempo absortos de la realidad, asimismo, un $91.89 \%$ de ellos afirman que se sintieron conmocionados y aturdidos durante un tiempo prolongado. Por otro lado, el $78.38 \%$ estaba convencido de que su hijo/a en realidad podía ver, pero era demasiado pequeño/a.

Este último aspecto se relaciona con el sentimiento de rechazo. $\mathrm{Al}$ respecto de este, cabe decir que el $77.78 \%$ de los padres y el $63.16 \%$ de las madres, antes de tener a su bebé, al ver a otros niños/as con discapacidad visual -especialmente en los casos más severos- pensaron en que, si pudieran, decidirían no tener un hijo/a con tales características. Por otro lado, el 78.38\% afirma que se negó a ver la realidad que estaba viviendo, frente a un 21.62\% que negó esta afirmación. Además, un $91.89 \%$ afirmó no haber sentido un rechazo hacia su hijo/a por el hecho de poseer una discapacidad visual.

En cuanto a los sentimientos de pena. De los 37 progenitores estudiados, 34 sintieron pena alguna vez hacia los hijos/as de otras personas por el hecho de tener una discapacidad, no obstante, este número desciende hasta los 28 cuando se trata del propio hijo/a. Del mismo modo, un $91.89 \%$ de ellos/as afirma haber sentido una profunda tristeza y pena por tener un hijo/a con discapacidad visual. Como dato destacado, más de un $20 \%$ de las madres tuvo episodios depresivos tras conocer el diagnóstico de su hijo/a.

En cuanto a la culpa se observa que las madres presentan índices ligeramente más elevados de culpabilidad hacia sí mismas que los padres (un 42.11\% frente a un 38.89\%). No obstante, el $22.22 \%$ de los hombres ha culpabilizado en alguna ocasión a su pareja de la discapacidad visual de su hijo/a, mientras que este porcentaje en las mujeres es del 15.79\%. Así, en global, un 40.54\% de los progenitores se ha culpado a sí mismo/a y un $18.92 \%$ a su pareja.

Estudiando los actos de agresividad un $18.92 \%$ de los progenitores ha manifestado conductas agresivas hacia sus amistades; un $24.32 \%$ hacia sus otros hijos/as; y un $27.03 \%$ hacia otros familiares. Además, uno de cada tres padres/madres ha dudado de la capacidad profesional de los médicos/as al diagnosticar la discapacidad visual y uno de cada cuatro mostró conductas agresivas hacia los/as profesionales que le atendieron.

El gráfico 1y 2 muestran, respectivamente, la carga emocional de los progenitores y la crisis percibida a raíz del nacimiento de un hijo con discapacidad visual 
Gráfico 1. Carga emocional e instrumental percibida por los progenitores a raíz de la discapacidad visual de su bijo/a.

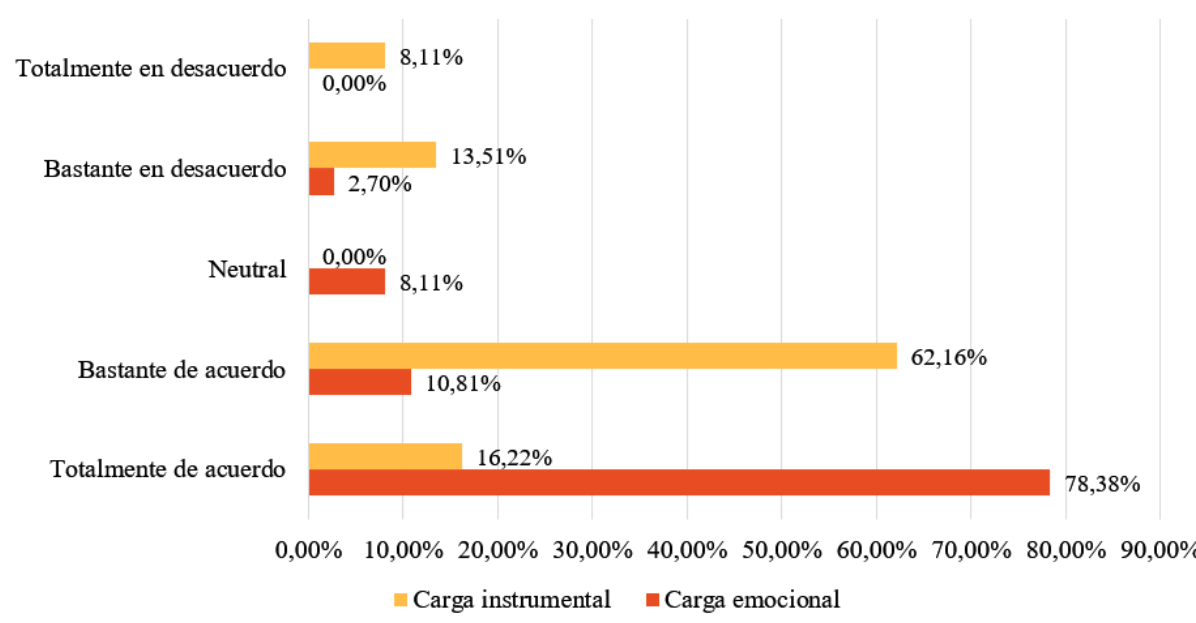

Gráfico 2. Crisis percibida por los progenitores a raíz de la discapacidad visual de su hijo/ a y recursos que han creído tener para afrontarlo.

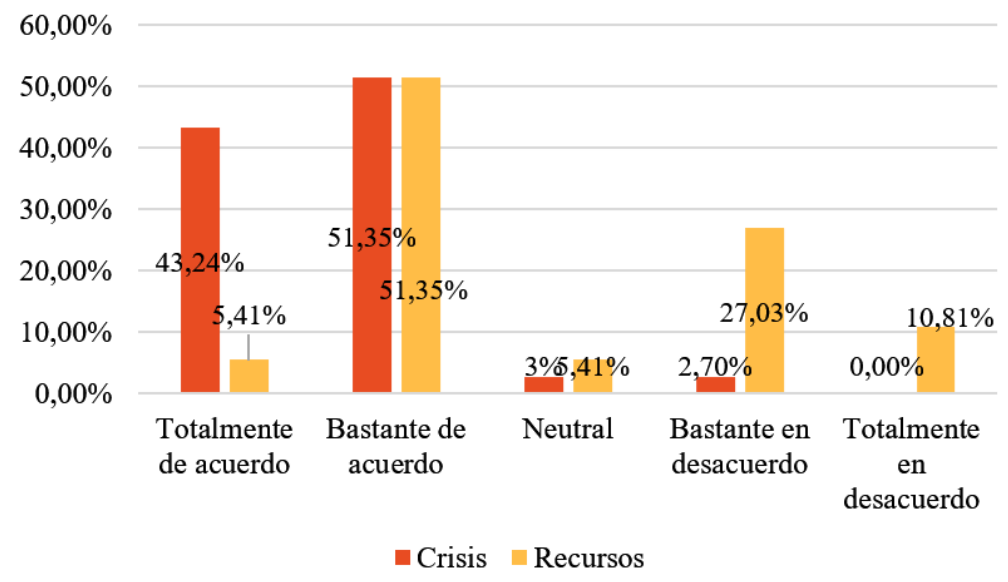

Se han estudiado también las expectativas que los progenitores tenían antes de tener a su bebé. Todos los padres/madres están totalmente de acuerdo en que antes de que naciera se habían imaginado cómo sería su hijo/a, pero solo un 13.51\% está totalmente de acuerdo con que había pensado en el hecho de que su hijo/a tuviera una discapacidad visual. Según el estudio realizado, ante la afirmación de que "las expectativas sobre mi hijo/a eran muy altas (buenas)", un 43.24\% de los progenitores estaba totalmente de acuerdo, un $2.70 \%$ bastante en desacuerdo y un $0 \%$ totalmente en desacuerdo.

Igualmente, gracias a una escala de Guttman, se han estudiado las diferentes áreas familiares en las que ha incidido el nacimiento de un hijo/a con discapacidad visual.

El nacimiento de un hijo/a con discapacidad visual ha incidido en la situación laboral de un $54.05 \%$ de los progenitores; en la situación sentimental de un 31.58\%; en la organización familiar de un $81.08 \%$; en las relaciones familiares de un $21.62 \%$; en las relaciones con los amigos y conocidos de un $72.97 \%$; en el ocio y tiempo libre de un $97.30 \%$; y en los ingresos económicos de un $91.89 \%$.

En cuanto al área económica, un $21.62 \%$ de los progenitores afirma que abandonaron su empleo de manera temporal para poder así dedicarle más tiempo a sus hijos/as, lo que influyó en la economía 
del hogar. No obstante, pese a que un $78.38 \%$ de los padres/madres decidieron no abandonar su empleo, el $91.89 \%$ afirman que la economía del hogar se vio resentida a raíz del nacimiento del niño/a con discapacidad visual, al aumentar los de desplazamientos a distintos especialistas y/o a las ayudas ópticas requeridas.

Por otro lado, en cuanto a las relaciones sexuales y la reproducción, un $27.03 \%$ de los progenitores vio disminuida la frecuencia con la que tenían relaciones sexuales y un $35.14 \%$ decidieron no tener más hijos/as a raíz de haber tenido uno/a con discapacidad visual. No obstante, aunque fueron un $35.14 \%$ los que decidieron no tener más hijos/as, el pensamiento de no tenerlos pasó por la mente de un $59.46 \%$ de los progenitores

También se ha estudiado el área social, habiendo observado que 19 progenitores se alejaron temporalmente se de sus amigos para poder dedicarle más tiempo a su hijo/a con discapacidad visual, mientras que fueron 6 los que se alejaron de sus familias por dicho motivo. Por otro lado, 8 progenitores decidieron alejarse de sus amigos puesto que no sentían comprensión por su parte, cifra que desciende hasta los 2 progenitores en el caso del alejamiento de los familiares por este motivo.

\section{IV.2. Hermanos}

En cuanto a los comportamientos de búsqueda de atención, cabe decir que un 33.33\% de los hermanos/as nunca han fingido dolores a propósito para llamar la atención de sus progenitores (dolores psicosomáticos), pero un $20 \%$ lo ha hecho muchas veces y un $20 \%$ lo ha hecho alguna vez. Igualmente, 5 de los 15 hermanos/as siempre han intentado desviar la atención de sus padres/madres provocando rabietas ya que sentía que no le hacían caso. Por un lado, un $26.67 \%$ de los hermanos/as ha sentido que alguna vez sus padres/madres le han abandonado por prestar más atención a su hermano/a con discapacidad visual; un $13.33 \%$ ha sentido que esto ha sido así siempre; y un $13.33 \%$ ha sentido que esto no ha sido así nunca. Además, se han registrado, por término medio, 4 casos en los que los hermanos/as han llevado a cabo comportamientos destructivos muchas veces y otros 4 en los que se ha realizado rara vez.

En cuanto a los comportamientos de rivalidad, un 6.67\% de los hermanos/as siempre ha mostrado conductas agresivas hacia su hermano/a con discapacidad visual; comportamiento que se ha repetido muchas veces en el $66.67 \%$ de los casos y rara vez en el $6.67 \%$. Con respecto a la agresividad hacia los progenitores, estos niveles porcentuales disminuyen, habiéndose registrado un $33.33 \%$ de los casos en los que nunca se han dado este tipo de conductas; y un $20 \%$ en los que se han dado rara vez. Abiertamente, 4 de los 15 hermanos/as estudiados han afirmado que siempre han sentido a su hermano/a con discapacidad como un rival por el cariño de sus padres/madres; 3 han tenido este sentimiento alguna vez y 3 nunca. Atendiendo a los celos, destaca el dato de que únicamente un $20 \%$ han afirmado no haber tenido celos de su hermano/a con discapacidad visual, mientras que un $80 \%$ sí los ha tenido.

Por otro lado, un $80 \%$ de los encuestados afirma que sus padres/madres muchas veces no han sabido o podido prestarle la misma atención que a su hermano/a con discapacidad, respecto a ello, llama especialmente la atención que ningún hermano/a ha afirmado de manera rotunda lo contrario. Además, un $33.33 \%$ de los hermanos / as, al ver que no solucionaba nada con otros comportamientos, ha decidido imitar las conductas de sus padres/madres y empezó a mostrar preocupación hacia su hermano/a con discapacidad visual en muchas ocasiones. Esta conducta abnegada ha sido manifestada rara vez por un $20 \%$ de los hermanos/as; alguna vez por un $13.33 \%$; y siempre por un $20 \%$. 


\section{Discusión}

Las familias antes el nacimiento de un bebé experimentan un cambio muy significativo en sus vidas y cuando el niño/a nace con discapacidad visual, las familias se sumergen en un proceso que, es duro, aunque también va acompañado de momentos de felicidad. Ser madre/padre no es fácil, y los niños/as no vienen con un manual de instrucciones. La conmoción, la culpa, la pena o la depresión son algunas de las emociones encontradas en los progenitores ante el nacimiento de un niño/a con discapacidad visual. Por su parte, los hermanos/as también tienen un papel importante en este asunto. Estos/as han manifestado que, en general, se han sentido desplazados/as en la familia, perdiendo "importancia" con respecto a su hermano/a con discapacidad. Esto ha llegado a provocar rabietas, dolores psicosomáticos, celos o rivalidad entre ellos/as. Además, es importante recalcar que, según las familias, las intervenciones llevadas a cabo desde el Trabajo Social en su situación no han sido del todo satisfactorias, pues han echado de menos, por lo general, un apoyo más emocional y no tanto económico, aunque este último no se rechaza, pues la economía de las familias, junto con otras áreas como la social o la familiar pueden verse severamente afectadas a raíz del nacimiento de un niño/a con discapacidad visual.

Derivado del rol especialmente protector de las familias hacia las personas más dependientes, cuando un hijo/a tiene una discapacidad visual, los progenitores, pese a tratar de mantener un equilibrio, pueden disminuir el tiempo y la energía empleada con el hijo/a sin discapacidad (Segura y Torras, 2014). Ante esta situación, los hermanos/as pueden reaccionar principalmente de dos modos:

Uno es el que señala Mendoza (2005), que surge cuando los hermanos/as de los niños/as con discapacidad tratan de llamar la atención de los progenitores, una de las acciones que pueden llevar a cabo es el comportamiento destructivo. Y el otro es el que señalan Segura y Torras (2014) cuando afirman que pueden aparecer las típicas rabietas y los pequeños hurtos en el intento de llamar la atención de los progenitores. A diferencia del aspecto anterior, en este sí se han obtenido resultados bastante elevados, pues la mayoría de los hermanos/as (55.33\%) lo han hecho.

Por otro lado, ante la desatención percibida por los hermanos/as, se puede crear un sistema de rivalidad (Lucerga y Sanz, 2004), en el que pueden aparecer diversos sentimientos y conductas. Según el estudio realizado, un $66.67 \%$ de los hermanos/as han tenido sentimientos de rivalidad hacia su hermano/a con discapacidad, frente a un $33.33 \%$ que no. Además, un $80 \%$ de los hermanos/as ha sentido una desatención por parte de sus progenitores en muchas ocasiones.

Según Stoneman (2006) y Lucerga y Sanz (2004), suelen aparecer celos entre ambos hermanos/as, sentimientos que nunca han sentido el $20 \%$ de los hermanos/as encuestados, pero que siempre han tenido el $33.33 \%$.

Segura y Torras (2014) sostienen que la conducta abnegada también es frecuente entre este colectivo. Los resultados obtenidos vislumbran que un 33.33\% de los hermanos/as ha tenido estas conductas muchas veces, frente a un $13.33 \%$ que no las ha tenido nunca.

Las conductas agresivas, estudiadas por Lucerga y Sanz (2004), se han observado con más frecuencia hacia los propios hermanos/as que hacia los progenitores. Así, mientras que un 86,67\% de los hermanos/as ha mostrado muchas o algunas veces conductas agresivas hacia sus hermanos/as, este porcentaje disminuye hasta el $46,66 \%$ en el caso de la agresividad hacia los padres/madres.

En el caso de los padres y madres, el primer aspecto que debe destacarse son las expectativas previas al nacimiento del bebé. A raíz del estudio realizado, y coincidiendo con autores como P. Ben 
Soussan (1999), citado en Díaz, 2004; Conti (2016) o Clavijo, Córdoba y Mora (2016), entre otros, puede concluirse que, cuando una pareja espera a un bebé, esta se forma una idea del niño/a esperado. Ahora bien, según Kew (1978), cuanto mayor sean esas expectativas, mayor será el impacto que tendrá en la familia el nacimiento de un niño/a con discapacidad visual. A este respecto cabe decir que, de acuerdo con el estudio, solo un $2.7 \%$ de los padres/madres no poseía altas expectativas sobre su hijo/a, mientras que un $43.24 \%$ sí. Este mismo $43.24 \%$ está, además, totalmente de acuerdo con que el nacimiento de este hijo/a pudo ser el causante de una crisis en la familia.

Díaz (2004) afirma que el proceso de adaptación y aceptación de un hijo/a con discapacidad visual consta de varios estadios o etapas en los que se encuentran diferentes sentimientos.

Atendiendo al rechazo se han estudiado tanto el rechazo al propio hijo/a, como hacia la situación y hacia los hijos/as ajenos. Clavijo, Córdoba y Mora (2016) están de acuerdo con el rechazo que algunos progenitores sienten hacia sus hijos/as y la colisión de este sentimiento con el de sobreprotección. No obstante, en el estudio se ha constatado que un $91.89 \%$ de los padres/madres no ha tenido sentimientos de rechazo hacia su hijo/a por el hecho de tener una discapacidad. Esto contrasta con los resultados obtenidos sobre el rechazo hacia los hijos/as con discapacidad visual de los demás progenitores, pues un $70.72 \%$ de los padres/madres encuestados afirman haber deseado no tener un hijo/a de tales características.

Este fenómeno ocurre también en el estudio de los sentimientos de pena hacia los niños/as, pues los padres y madres han manifestado índices más altos al hablar sobre los hijos/as ajenos (34 frente a 28 casos). En cuanto a la pena por el hecho de haber tenido un hijo/a con discapacidad visual, el $91.89 \%$ de los padres/madres afirman que es un sentimiento conocido por ellos, como sentencia Olshansky (1972). Por otro lado, cabe destacar también que un $21.05 \%$ de las madres tuvo episodios depresivos, posibilidad expresada por Leonhardt (2008).

Estos sentimientos pueden finalmente desembocar en crisis familiares y repercutir en áreas tan importantes como la sentimental, la social, laboral o la familiar. Es posible que, con una intervención adecuada por parte de los/as trabajadores/as sociales que atienden a este tipo de familias, la repercusión de la discapacidad no sea tan impactante.

En cuanto a las intervenciones llevadas a cabo desde el Trabajo Social con estas familias, se ha visto que hay un deseo de cambio generalizado (89.47\%) por parte de los encuestados. Según estos, un $94.74 \%$ de las intervenciones de las que han sido partícipes siempre han sido con el objetivo de tratar con el niño/ a con discapacidad, sin sentir a la propia familia como un objeto de intervención y atención.

\section{Conclusiones}

La familia es el primer entorno en el que la persona se desarrolla. La relación con los progenitores es fundamental como núcleo de socialización y aprendizaje, y la relación con los hermanos/as lo es para que se produzca una buena interacción en la que los hermanos/as aprendan a compartir, a demostrar lealtad, a rivalizar, etc. Así, la discapacidad de uno de los miembros no puede ser tomada como una característica individual, sino como un fenómeno social que repercute especialmente en una parte del entorno o, según el modelo sistémico, en una parte de un sistema: la familia.

Tanto en el área de la discapacidad como en la de las familias en conflicto, el trabajador/a social tiene un papel especialmente relevante y una función con gran peso. No obstante, cuando 
ambos ejes de intervención confluyen en una misma realidad, las intervenciones pueden tomar un enfoque concéntrico priorizando la atención más hacia la discapacidad que a la persona. Esto puede conllevar la desatención de las necesidades específicas de la familia y, en consecuencia, una intervención global insatisfactoria en la que se desaprovechan las potenciales mejoras en la calidad de vida de todo el conjunto familiar.

Así, pese a que se ha avanzado mucho en los modelos de atención a las personas con discapacidad, se considera necesario dar un paso más, un salto cualitativo que incluya a las familias como receptoras de atención y apoyo específico por parte del Trabajo Social, visibilizando situaciones y necesidades reales de estas familias, donde el trabajo colaborativo y cooperativo entre los padres/madres y los profesionales es esencial. Los primeros deben formar parte activa del proceso de intervención y no ser meramente receptores pasivos de atención, cobrando especial relevancia la relación de ayuda con el profesional, en la que ambas partes entablen una relación de confianza mutua que permita mejorar las intervenciones llevadas a cabo. La responsabilidad de la creación de esta relación no reside únicamente en el/la trabajador/a social, sino también en la familia.

\section{Referencias bibliográficas}

CONFEDERACION ESPAÑOLA DE ORGANIZACIONES EN FAVOR DE LAS PERSONAS CON DISCAPACIDAD INTELECTUAL. (2006). Libro de Ponencias del III Congreso Nacional de Familias con Personas con Discapacidad Intelectual. Zaragoza, España: Confederación Española de Organizaciones en favor de las Personas con Discapacidad Intelectual . Recuperado el 27 de Junio de 2019, de http://www.dincat.cat/iii-congresonacional-de-familias-de-personas-con-discapacidad-intelectual_21224.pdf

DEL FRESNO, M., y SEGADO, S. (2013). Trabajo Social con familias: los estilos familiares como indicadores de riesgos, una investigación etnográfica. Portularia, XIII(1), 37-46. http://doi.org/10.5218/prts.2013.0005

DIAZ, R. M. (2004). Personas con discapacidad: Una aproximación desde el Trabajo Social. Sevilla: Aconcagua Libros.

EGEA, C., y SARABIA, A. (2004). Visión y modelos conceptuales de la discapacidad. Polibea, 73, 2942. Recuperado el 26 de Junio de 2019, de https://sid.usal.es/7589/8-2-6 1

FREIXA, M. (1993). Familia y deficiencia mental. Realidad, necesidades y recursos de los hermanos. Salamanca: Amarú. Recuperado de https://www.tdx.cat/bitstream/handle/10803/2361/03.MFN_3de8.pdf

GRAD, J., y TIZARD, J. (1961). Los discapacitados mentales y sus familias. Inglaterra: Oxford University Press.

HOLT, K. (junio de 1958). La influencia de un niño con deficiencia intelectual sobre las limitaciones familiares. Revista de investigación de la deficiencia mental, II(1), 28-36. https://doi.org/10.1111/j.1365-2788.1958.tb00383.x

INSTITUTO DE MAYORES Y SERVICIOS SOCIALES; Ministerio de Trabajo y Asuntos Sociales; y Secretaría de Estado de Servicios Sociales, Familias y Discapacidad. (2004). Recursos para la atención de personas menores de 65 años en situación de dependencia. En IMSERSO, M. d. Sociales, y F. y. y Secretaría de Estado de Servicios Sociales, Atención a las personas en situación de dependencia en España. Libro Blanco (págs. 4-111). Madrid, España: 
Gobierno de España. Recuperado el 14 de Marzo de 2019, de https://www.uab.cat/Document/580/416/LibroBlancoDependencia_01,0.pdf

KEW, S. (1978). Los demás hermanos de la familia: Minusvalia y crisis familiar. (J. Fernández, Trad.) Madrid, España: SEREM, Servicio de Publicaciones, Ministerio de Sanidad y Seguridad Social.

LEONHARDTt, M. (2008). Primeros sentimientos y emociones que experimentan los padres de los niños con ceguera o baja visión. En C. García-Trevijano, M. Leonhardt, B. Oyarzábal, y I. Vecilla, Construir juntos espacios de esperanza. Orientaciones para el profesional de atención temprana a niños con ceguera o deficiencia visual (Primera ed., págs. 57-77). Madrid, España: ONCE.

LORENZO, J. (2015). El papel de las familias de las personas con discapacidad intelectual en los procesos de intervención social, ante la comisión de un delito. Una mirada desde el Trabajo Social. Alicante, España: Universidad de Alicante. Recuperado el 26 de Junio de 2019, de https://rua.ua.es/dspace/bitstream/10045/77529/1/tesis_josefa_lorenzo_garcia.pdf

LUCERGA, R. M., y SANZ, M. J. (2004). Puentes invisibles: El desarrollo emocional de los niños con discapacidad visual grave. Madrid, España: ONCE, Dirección de Educación.

MENDOZA, C. (2005). El hermano del niño enfermo crónico: especial e invisible. Santiago, Chile: Tesis pregrado. Universidad Academia de Humanismo Cristiano. Recuperado el 30 de Mayo de 2019, de http://bibliotecadigital.academia.cl/xmlui/handle/123456789/2523

MUYOR, J. (2011). La conciencia del trabajo social en la discapacidad: hacia un modelo de intervención social basado en derechos. Documentos de Trabajo Social: Revista de trabajo y acción social(49), 9-33. Recuperado el 26 de Junio de 2019, de https: / / dialnet.unirioja.es/servlet/articulo?codigo $=4111301$

OLSHANSKY, S. (1962). Dolor crónico: una respuesta a tener un hijo con deficiencia mental. Trabajo social, 43(4), 190-193. http://doi.org/10.1177/104438946204300404

ORGANIZACIÓN NACIONAL DE CIEGOS ESPAÑOLES (2019). Datos de afiliados a la ONCE. Recuperado el 5 de Marzo de 2019, de Grupo Social ONCE: https://www.once.es/dejanosayudarte/afiliacion/datos-de-afiliados-a-la-once

PALACIOS, A. (2008). El modelo social de discapacidad: origenes, caracterización y plasmación en la Convención Internacional sobre los Derechos de las Personas con Discapacidad. Madrid: Cinca, CERMI. Recuperado el 24 de Junio de 2019, de https://www.cermi.es/sites/default/files/docs/ colecciones/Elmodelosocialdediscapacidad.pdf

PLENA INCLUSIÓN. (2017). Modelo de Atención Sociofamiliar en Centros de Atención Temprana desde la perspectiva del Trabajo Social (Información, orientación y apoyo familiar). Madrid: Plena Inclusión Madrid. Recuperado el 18 de Junio de 2019, de https://plenainclusionmadrid.org/wpcontent/uploads/2017/12/Modelo-ASF-ONLINE.pdf

PONCE, À. (2008). Apoyando a los hermanos. Tres propuestas de intervencion con hermanos de personas con discapacidad intelectual. Madrid: FEAPS. Recuperado el 2 de Junio de 2019, de https://angelsponce.com/wp-content/uploads/2018/03/apoyando_hermanos.pdf

SEGURA, A. M., y TORRAS, A. (2014). El otro hermano. Riberdis(14), 30-35. Recuperado el 21 de Mayo de 2019, de http://riberdis.cedd.net/bitstream/handle/11181/4354/El\%20otro\%20hermano.pdf

STONEMAN, Z. (2006). Hermanos de niños con discapacidad: temas de investigación. Siglo Cero, 37(219), 13-30. Recuperado el 2 de Junio de 2019, de http://sid.usal.es/idocs/F8/ART9089/articulos2.pdf 